\title{
Proteção contra corrosão da superfície do aço 1020 contendo polipirrol depositado por cronoamperometria em meio de ácido metanossulfônico
}

Corrosion protection of 1020 steel surface containing polypyrrole deposited by chronoamperometry in methanesulfonic acid medium

Alex Fernandes de Souza1', Liu Yao Cho², Andrea Santos Liu

\section{RESUMO}

O polipirrol (PPy) é um polímero condutor que tem sido investigado para proteger metais oxidáveis contra corrosão. A utilização deste polímero agrega algumas vantagens, destacando-se a baixa toxicidade, estabilidade química e facilidade de síntese química ou eletroquímica. Neste trabalho, o filme de polipirrol foi depositado eletroquimicamente por cronoamperometria sobre o aço 1020, em meio aquoso contendo ácido metanossulfônico. O resultado do ensaio de polarização potenciodinâmica em meio agressivo de cloreto mostrou que o potencial de corrosão da superfície de aço revestida com o polímero foi deslocado para a direção mais positiva em relação ao metal apenas polido, indicando proteção anódica. Além disso, a morfologia investigada por Microscopia Eletrônica de Varredura (MEV) indicou a formação de filme de PPy compacto e homogêneo sobre a superfície do aço.

Palavras-Chave: Polipirrol, Aço 1020, Eletrodeposição.

\section{ABSTRACT}

Polypyrrole (PPy) is a conducting polymer that has been investigated as a corrosion inhibitor for oxidizable metals. The use of this polymer has some advantages, such as low toxicity, chemical stability, and the possibility of being either chemical or electrochemical synthetized. In this work the polypyrrole film was electrodeposited by chronoamperometry in methanesulfonic acid. The result of the potentiodynamic polarization test in aggressive sodium chloride medium has shown that the corrosion potentia of metal coated by PPy was displaced to a more positive value in relation to the polished metal, indicating anodic protection. Furthermore, the Scanning Electron Microscopy (SEM) indicated the formation of compact and homogeneous PPy film on the stee surface.

Keywords: Polypyrrole, 1020 steel, Electrodeposition.

\footnotetext{
${ }^{1}$ Instituto Federal de Educação, Ciência e Tecnologia de São Paulo - Coordenadoria de Química - São José dos Campos/SP - Brasil. ${ }^{2}$ Universidade do Vale do Paraíba - Faculdade de Engenharias, Arquitetura e Urbanismo - Instituto de Pesquisa e Desenvolvimento - São José dos Campos/SP - Brasil.

Autor correspondente: Alex Fernandes de Souza | Instituto Federal de Educação, Ciência e Tecnologia de São Paulo - Química | Rodovia Presidente Dutra, saída km 145 | CEP 12.223-201 - São José dos Campos/SP - Brasil | E-mail: alex_fernandes1989@live.com

Recebido: 14 Fev. 2018 | Aceito: 23 Mar. 2018
} 


\section{INTRODUÇÃO}

O aço é um metal largamente utilizado no setor industrial, formado principalmente por ferro e carbono. As propriedades mecânicas do aço diferem na medida em que se varia o teor de carbono em sua composição, podendo ser de $0,2 \%$ até $1,8 \%$. Atualmente, o aço 1020 é um dos materiais mais utilizados devido à sua baixa temperabilidade, excelente forjabilidade, baixo custo, ductilidade e maior resistência à tração. Apresenta inúmeras aplicações, como fabricação de parafusos, fixadores, eixos e engrenagens, sendo estes componentes altamente importantes no funcionamento seguro de uma máquina ${ }^{1}$.

Os processos de desgaste e corrosão do aço 1020 envolvem mecanismos eletroquímicos e ações combinadas que muitas vezes resultam em um aumento significativo na degradação do material. As interações entre abrasão, fricção, impacto e corrosão podem aumentar significativamente a perda total de material, especialmente em um ambiente aquoso, produzindo assim um efeito sinérgico. O efeito sinérgico é bastante pronunciado para os aços carbono ${ }^{2}$. Este efeito é o resultado de uma ação mecânica e eletroquímica e é caracterizado pela influência eletroquímica na deformação plástica nas propriedades do metal, que ocorre mais comumente em um ambiente composto de ação mecânica, causando deformação, e um agente corrosivo ${ }^{3}$.

Atualmente a proteção contra corrosão do aço é realizada por diferentes tratamentos superficiais, ressaltando-se a fosfatização e a cromatização. As composições básicas de soluções fosfatizantes necessitam de catalisadores que utilizam metais pesados como níquel (Ni) e cobre $(\mathrm{Cu})$ em sua composição, além disso, contêm compostos a base de fósforo, que causam a eutrofização de recursos hídricos, quando descartados de forma inadequada. Por outro lado, os compostos a base de cromo hexavalente são tóxicos e carcinogênicos. Desta forma, grupos de pesquisa buscam alternativas aos processos de fosfatização e à utilização de $\mathrm{Cr}^{+6}$, destacando como substitutos os polímeros condutores ${ }^{4}$.

Polímeros condutores, como polipirrol (PPy) e polianilina (PAni), têm uma vasta gama de aplicações no seu estado dopado ou parcialmente oxidado, e apresentam resultados interessantes no efeito da proteção contra corrosão do aç̧ ${ }^{5}$. Estes polímeros são eletricamente condutores e podem ser denominados revestimentos ativos, pois podem ocorrer interações químicas ou eletroquímicas com metais ativos ${ }^{6}$.

A síntese dos filmes de PPy pode ser realizada química ou eletroquimicamente, em meio aquoso ou orgânico. Os filmes de PPy são vantajosos pois apresentam boas propriedades mecânicas, baixa rugosidade, flexibilidade e baixo custo de produção. Além dessas propriedades, o PPy se destaca pela sua estabilidade química e alta condutividade elétrica, quando dopado. Todas essas características associadas à baixa toxicidade tornam este polímero vantajoso na atuação para proteção contra corrosão de metais oxidáveis, como o aço $1020^{7}$.
A polimerização eletroquímica do pirrol em eletrodos de aço tem sido retratada como complicada devido à oxidação do metal, que concorre simultaneamente com a oxidação do monômero, podendo inibir o crescimento do polímero ou reduzir a aderência do polímero ao metal ${ }^{8}$. Neste processo eletroquímico, a seleção do eletrólito é fundamental para evitar que o mesmo promova a oxidação do substrato metálico?.

Estudos prévios desenvolvidos em nosso laboratório têm mostrado que filmes homogêneos foram formados eletroquimicamente em superfícies de alumínio quando se utilizou ácido sulfônico como eletrólito ${ }^{10,11}$.

Neste trabalho foi investigada a eletrodeposição do PPy sobre a superfície do aço $1020 \mathrm{em}$ meio aquoso contendo ácido metanossulfônico. O desempenho do filme na proteção do metal contra corrosão também foi analisado através de ensaios de polarização potenciodinâmica em meio aquoso contendo íons de cloreto.

\section{MATERIAIS E MÉTODOS}

Para os ensaios eletroquímicos, utilizou-se um Potenciostato/ Galvanostato modelo MQPG-01 da Microquímica, conectado e controlado por um microcomputador. Os testes foram realizados em uma célula eletrolítica contendo três eletrodos: eletrodo de trabalho (aço 1020 embutido em teflon e com área exposta de $\left.0,53 \mathrm{~cm}^{2}\right)$; eletrodo de referência $(\mathrm{Ag} / \mathrm{AgCl})$; e eletrodo auxiliar (bastão de platina). A composição do metal de aço 1020 é apresentada na Tabela 1.

Tabela : Composição química do aço carbono ABNT 1020 (\%).

\begin{tabular}{c|c|c|c|c} 
Elemento & $\mathbf{C}$ & $\mathbf{M n}$ & $\mathbf{P}$ & $\mathbf{S}$ \\
\hline Composição & $0,18 / 0,23$ & $0,30 / 0,600$ & 0,04 & 0,05 \\
\hline
\end{tabular}

Antes de cada ensaio, a superfície do aço foi desbastada com lixas d'água de granulometria 220, 400, 600 e 1200 mesh, respectivamente. Na sequência, o eletrodo de aço foi enxaguado com água destilada.

A eletrodeposição do PPy foi realizada por cronoamperometria, aplicando-se o potencial de 1,2 V vs $\mathrm{AgCl} / \mathrm{Cl}$ durante $30 \mathrm{~min}$. Uma solução aquosa contendo $0,2 \mathrm{~mol} \cdot \mathrm{L}^{-1}$ de ácido metanossulfônico e $0,1 \mathrm{~mol} \cdot \mathrm{L}^{-1}$ de pirrol foi utilizada neste processo.

A morfologia da superfície revestida com filme de PPy foi analisada por Microscopia Eletrônica de Varredura (MEV) utilizando o equipamento Jeol JXA-840A. A rugosidade do filme foi analisada por meio da Microscopia de Força Atômica (MAF) utilizando o equipamento SPM- $9500 \mathrm{J3}$.

A proteção contra corrosão da superfície do aço recoberta pelo filme de PPy foi investigada por ensaios de polarização potenciodinâmica, variando o potencial de $-1,0$ a $1,5 \mathrm{~V}$ a $2,0 \mathrm{mV} \cdot \mathrm{s}^{-1}$, em solução de $\mathrm{NaCl} 3,5 \%(\mathrm{~m} / \mathrm{v})$ e $\mathrm{pH}=6,4$. 


\section{RESULTADO E DISCUSSÃO}

Estudos eletroquímicos prévios mostraram que o filme de PPy é depositado no eletrodo de aço através de ensaios de voltametria cíclica. Foi observado que as correntes anódicas começaram a aumentar a partir de $+1,0 \mathrm{~V}$ vs $\mathrm{Ag} / \mathrm{AgCl}$, indicando a oxidação do monômero e deposição do filme polimérico ${ }^{12}$.

No presente trabalho, a eletrodeposição do filme de PPy foi realizada por ensaios de cronoamperometria. Foi observado que a aplicação de 1,0 V vs $\mathrm{Ag} / \mathrm{AgCl}$ não foi suficiente para recobrir completamente a superfície de aço pelo filme polimérico. Posteriormente, aplicou-se 1,2 V durante 30 minutos, que resultou na formação de um filme preto homogêneo e que recobriu completamente o eletrodo de trabalho. A Fig. 1 apresenta a curva de corrente vs tempo obtido neste ensaio.

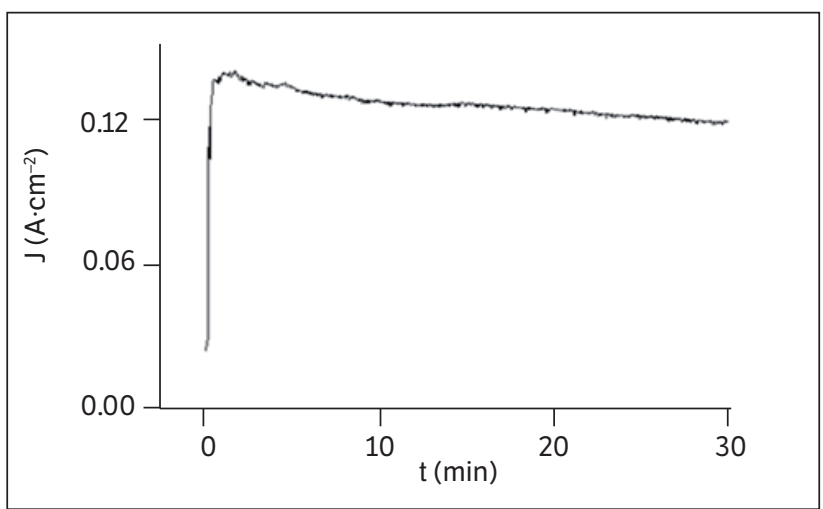

Figura 1: Crescimento do filme de PPy aplicando-se 1,2 V.

Observa-se na Fig. 1 que, logo após a aplicação do potencial de $1,2 \mathrm{~V}$, o valor da corrente aumentou e se manteve numa faixa de corrente de aproximadamente $0,12 \mathrm{~A} \cdot \mathrm{cm}^{-2}$, indicando o crescimento do filme de PPy sobre a superfície do eletrodo de aço 1020, na forma condutora ${ }^{13}$.

Foram realizados ensaios de polarização potenciodinâmica em meio agressivo de $\mathrm{NaCl}$ 0,1 $\mathrm{mol} \cdot \mathrm{L}^{-1}$ com a finalidade de analisar o desempenho do filme de PPy contra corrosão. Na Fig. 2 são apresentadas as curvas de Tafel para estes ensaios.



Figura 2: Curva de Tafel para as superfícies do aço: (1) polida e (2) recoberta com PPy.
O potencial de corrosão do aço apenas polido (1) foi $-0,63 \mathrm{~V}$, enquanto o do aço revestido com a PPy (2) foi $-0,25 \mathrm{~V}$. O deslocamento do potencial de corrosão para a direção positiva indica proteção anódica pelo filme polimérico ${ }^{14}$.

Também foi observado na Fig. 2 que as densidades de correntes anódicas, associadas com a oxidação do metal, foram menores para o aço recoberto com o PPy. Se considerarmos os gráficos da Fig. 2, no potencial de $+0,5 \mathrm{~V}$ a densidade de corrente anódica para a superfície recoberta com PPy é $0,00169 \mathrm{~mA} \cdot \mathrm{cm}^{-2}$, enquanto que para a superfície apenas polida é $0,00891 \mathrm{~mA} \cdot \mathrm{cm}^{-2}$, o que também indica a proteção do metal pelo filme polimérico.

A morfologia do aço 1020 recoberto com o filme de PPy foi analisada por Microscopia Eletrônica de Varredura. A Fig. 3 apresenta o MEV para a superfície do aço polida e recoberta com o filme.

(A)

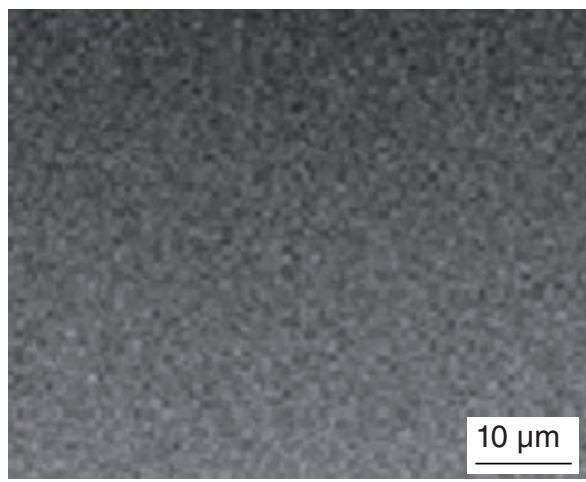

(B)

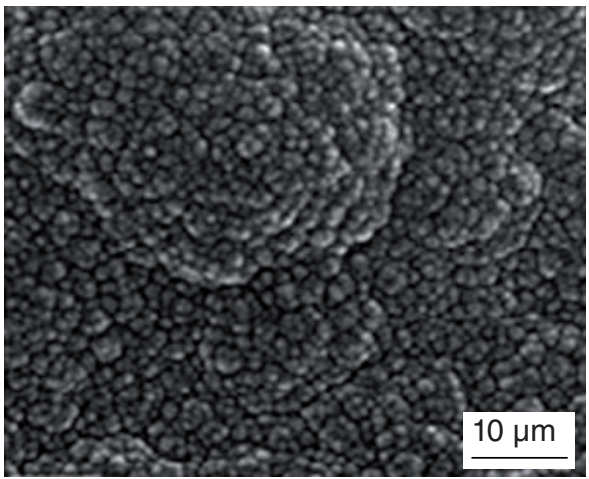

Figura 3: MEV para as superfícies do aço: (A) polida e (B) recoberta com PPy a 1,2 V.

A micrografia indicou que o filme de PPy eletrodepositado a $1,2 \mathrm{~V}$ recobriu totalmente a superfície do aço de maneira uniforme. Este resultado está coerente com que é apresentado na literatura, que relata a formação de filmes de PPy homogêneo e sem falhas sobre a superfície do metal inibindo, portanto, a interação química do aço com o meio agressivo ${ }^{15}$.

A rugosidade do filme foi investigada por meio de Microscopia de Força Atômica (MAF). A topografia do filme é apresentada na Fig. 4.

É possível visualizar que o filme de PPy não apresenta grandes variações ao longo da superfície sendo, portanto, uniforme e 
compacto, com valor de rugosidade média quadrática de 4,2 $\mu \mathrm{m}$. Ressalta-se que filmes mais compactos são mais resistentes à degradação ${ }^{8}$.

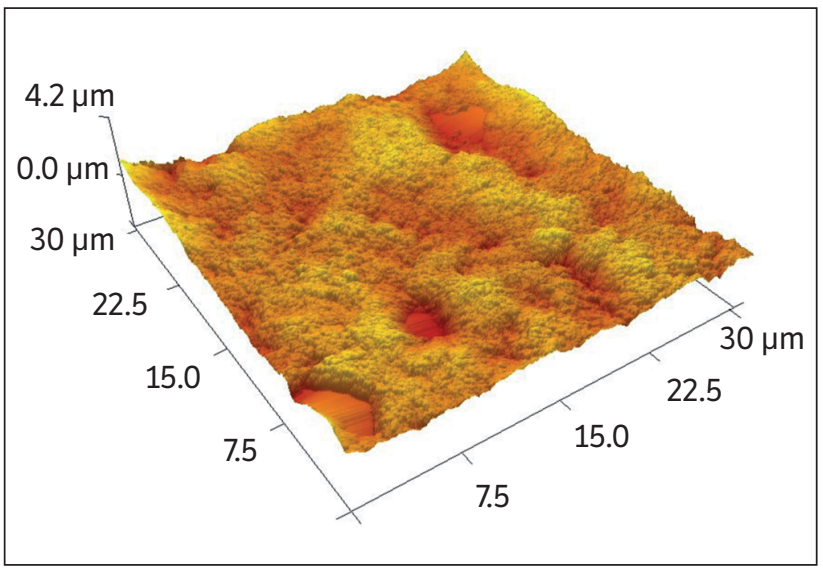

Figura 4: MAF do filme de PPy eletrodepositado sobre a superfície do aço 1020 .

Foi realizado um ensaio de imersão em meio corrosivo de cloreto, com a superfície do aço 1020 recoberta com o filme de PPy eletrodepositado a 1,2 V vs Ag/ $\mathrm{AgCl}$, durante 5 dias. Mesmo após esse período não foram observadas falhas no filme.

\section{CONCLUSÃO}

O estudo demonstrou que o filme de PPy é homogêneo, aderente e facilmente eletrodepositado por cronoamperometria sobre o aço 1020. O teste de corrosão em meio agressivo de cloreto de sódio mostrou que o filme é eficiente como inibidor de corrosão. Notou-se também que a camada polimérica foi aderente e se manteve estável mesmo após o ensaio de corrosão. A partir desses dados, conclui-se que o aço recoberto com o filme do polímero, enquanto íntegro e sem falhas, é menos suscetível à corrosão, em relação ao aço sem recobrimento, apresentando deslocamento de potencial de corrosão para valores mais positivos.

\section{REFERENCES}

1. Mercan S, Aydin S, Özdemir N. Effect of welding parameters on the fatigue properties of dissimilar AISI 2205-AISI 1020 joined friction welding. International Journal of Fatigue. 2015;81:78-90. Disponível em: https://doi.org/10.1016/j.ijfatigue.2015.07.023

2. Wang L, Qiu N, Hellman DH, Zhu X. An experimental study on cavitation erosion-corrosion performance of ANSI 1020 and ANSI 4135 steel. J Mech. Sci. Technol. 2016;30(2):533-539. Disponível em: https://doi.org/10.1007/s12206-016-0106-9

3. Simescu F, Idrissi $H$. Effect of zinc phosphate chemical conversion coating on corrosion behaviour of mild steel in alkaline medium: protection of rebars in reinforced concrete. Sci.
Technol. Adv. Mater. 2008;9(4):045009. Disponível em: http:// doi.org/10.1088/1468-6996/9/4/045009

4. Rios EC, Zimer AM, Pereira EC, Mascaro LH. Analysis of AISI 1020 steel corrosion in seawater by coupling electrochemical noise and optical microscopy. Electrochimica Acta. 2014;124:211-217. Disponível em: https://doi.org/10.1016/j. electacta.2013.10.059

5. Montoya P, Martins CR, Melo HG, Aoki IV, Jaramillo F, Calderón JA. Synthesis of polypyrrole-magnetite/silane coatings on steel and assessment of anticorrosive properties. Electrochimica Acta. 2014;124(1):100-108. Disponível em: https://doi.org/10.1016/j.electacta.2013.07.105

6. Rajagopalan R, Iroh JO. Characterization of polyanilinepolypyrrole composite coatings on low carbon steel: a XPS and infrared spectroscopy study. Applied Surface Science. 2003; 218(1-4):58-69. Disponível em: https://doi.org/10.1016/ S0169-4332(03)00579-8

7. Hermelin E, Petitjean J, Aeiyach S, Lacroix JC, Lacaze PC. Industrial polypyrrole electrodeposition on zinc electroplated steel. Journal of Applied Eletrochemistry. 2001;31(8): 905-911. Disponível em: https://doi.org/10.1023/A:1017550922578

8. El Jaouhari A, Laabd M, Bazzaoui EA, Albourine A, Martins JI, Wang R, et al. Electrochemical and spectroscopical studies of polypyrrole synthesized on carbon steel from aqueous medium. Synthetic Metals. 2015; 209:11-18. Disponível em: https://doi.org/10.1016/j.synthmet.2015.06.017

9. Hamer WJ, Koene L, de Wit JHW. Formation and electrochemical behaviour of poly(pyrrole) coatings on steel substrates. Materials and Corrosion. 2004;55(9):653-658. Disponível em: https://doi.org/10.1002/maco.200303778

10. Souza AF, Liu AS, Cho LY, Reis JL, Ferreira BR. Proteção contra corrosão do alumínio 2024-T3 por filme de polipirrol eletrodepositado em ácido p-tolueno sulfônico. Revista Brasileira de Aplicação de Vácuo. 2017; 36(1):34-38. Disponível em: http://dx.doi.org/10.17563/rbav.v36i1.1059

11. Liu AS, Bezerra KM, Cho LY. Electrodeposition of polypyrrole on aluminum alloy 2024-t3 from dodecylbenzenesulfonic acid medium. Inte. J. Recent Sci. Res. 2017;8(11):2144921454. Disponível em: http://dx.doi.org/10.24327/ijrsr.2017. 0811.1076

12. Souza AF, Cho LY, Liu AS. Corrosion protection of 1020 steel by conducting films electrodeposited in methanesulfonic acid. Journal of Experimental Techniques and Instrumentation. 2018;1(1):41-48. Disponível em: http://doi.org/10.30609/JETI. 2018-1.5303

13. Vera R, Schrebler R, Grez $\mathrm{P}$, Romero $\mathrm{H}$. The corrosioninhibiting effect of polypyrrole films doped with p-toluenesulfonate, benzene-sulfonate or dodecyl-sulfate anions, as coating on stainless steel in $\mathrm{NaCl}$ aqueous solutions. Progress in Organic Coatings. 2014; 77(4):853-858. Disponível em: https://doi.org/10.1016/j.porgcoat.2014.01.015

14. Herrasti P, Ocón P. Polypyrrole layers for steel protection. Applied Surface Science. 2001;172(3-4):276-284. Disponível em: https://doi.org/10.1016/S0169-4332(00)00866-7

15. Hussein MA, Al-Juaid SS, Abu-Zied BM, Hermas AEA. Electrodeposition and corrosion protection performance of polypyrrole composites on aluminum. Int. J. Electrochem. Sci. 2016;11:3938-3951. Disponível em: http://doi.org/10.20964/ 11049 